\title{
A COMPLEX VERSION OF THE BAER-KRULL THEOREMS
}

\author{
M.J. de la Puente* \\ Departamento de Algebra \\ Facultad de Matemáticas, Universidad Complutense, 28040-Madrid, Spain. \\ e-mail: MariaJesus_delaPuente@mat.ucm.es
}

\begin{abstract}
A bstract
In the setting of the complex spectrum of a field of characteristic zero we give complex analogs of the celebrated (real) Baer-Krull theorems relating the orderings of a field compatible with a given valuation ring with the orderings of the residue class field of that valuation ring.
\end{abstract}

\section{INTRODUCTION}

In the setting of the complex spectrum of a field of characteristic zero we want to give complex analogs of the celebrated (real) Baer-Krull theorems relating the orderings of a field compatible with a given valuation ring with the orderings of the residue class field of that valuation ring.

Recall that a field $K$ is called real if $K$ admits orderings. The set of such orderings is denoted $\operatorname{Spec}_{\mathrm{r}}(K)$ and called the real spectrum of $K$. Real fields are of zero characteristic.

If $B$ is a local ring, let $m_{B}, U_{B}$ and $\bar{K}:=B / m_{B}$ respectively denote the maximal ideal, the group of units and the residue class field of $B$. If $K$ is a field and $B \subseteq K$ is a valuation ring of $K$, then let $\lambda_{B}: K \rightarrow \bar{K} \cup\{\infty\}$, $\Gamma_{B}:=K \backslash\{0\} / U_{B}$ and $v_{B}: K \rightarrow \Gamma_{B} \cup\{\infty\}$ respectively denote the canonical place, the value group of $B$ (additively written) and the canonical valuation associated to $B$. The restriction of $\lambda_{B}$ to $B$ is the canonical residue class

1991 AMS subject classification: 12J10, 12J15.

* Partially supported by CICYT, PB 89/0379-C02-02 and by DGICYT 
morphism. Note that the characteristic of $\bar{K}$ is zero if and only if $B$ is finite over $\mathbb{Q}$, i.e., $\mathbb{Q}$ is a subring of $B$.

If $K$ is a field and $B$ is a valuation ring of $K$, recall that

(a) $B$ is called residually real if the residue class field $\bar{K}$ is real i.e., if Spec $_{\mathbf{r}}(\bar{K}) \neq \emptyset$.

(b) If $K$ is real and $\beta$ belongs to $\operatorname{Spec}_{\mathbf{r}}(K)$, then $B$ is called $\beta$-convex if for all $a \in K, b \in B$, the condition $a^{2} \leq_{\beta} b^{2}$ implies $a \in B$.

In the late 1920's and early 1930's it was proved -using a different language- by Baer and Krull (see $[\mathrm{Ba}]$ and $[\mathrm{Kr}]$ ) that:

(Ba-Kr 1) If $B$ is $\beta$-convex then there exists a unique $\bar{\beta}$ in $\operatorname{Spec}_{\mathrm{r}}(\bar{K})$ such that for all $x \in U_{B}, x>_{\beta} 0$ if and only if $\lambda_{B}(x)>_{\bar{\beta}} 0$. In particular, $B$ is residually real.

(Ba-Kr 2) If $B \subseteq K$ is residually real, then for every $\gamma \in \operatorname{Spec}_{\mathbf{r}}(\bar{K})$ there exists $\beta$ in $\operatorname{Spec}_{1}(K)$ such that for all $a, b \in B$, the condition $a \leq_{\beta} b$ implies $\lambda_{B}(a) \leq_{\gamma} \lambda_{B}(b)$. Moreover, $\gamma=\bar{\beta}$ and $B$ is $\beta$-convex.

Already considered in $[\mathrm{Kr}]$, the relationship between $\gamma$ and all possible $\beta$ satisfying ( $\mathrm{Ba}-\mathrm{Kr} 2)$ was completely elucidated in $[\mathrm{Br}]$. The notion of real place (the place associated to a residually real valuation ring) was introduced and studied in $[\mathrm{L}]$.

Today, we can deduce (Ba-Kr 1) and the second assertion in (Ba-Kr 2) from the well-known fact that the following statements are equivalent:

(i) $B$ is $\beta$-convex,

(ii) $m_{B}$ is $\beta$-convex in $B$,

(iii) there exists a unique $\bar{\beta}$ in $\operatorname{Spec}_{\mathrm{r}}(\bar{K})$ with respect to which $\left.\lambda_{B}\right|_{B}: B \rightarrow \bar{K}$ is an order-preserving ring morphism,

(iv) $B$ contains the valuation ring $\left\{x \in K: x^{2} \leq_{\beta} n^{2}\right.$, some $\left.n \in \mathbb{N}\right\}$,

(v) there exists $\gamma$ in $\operatorname{Spec}_{\mathrm{r}}(\bar{K})$ such that for all $a, b \in B$, the condition $a^{2} \leq_{\beta} b^{2}$ implies $\lambda_{B}\left(a^{2}\right) \leq_{\gamma} \lambda_{B}\left(b^{2}\right)$.

These are assertions about pushing down orderings from $K$ to $\bar{K}$. On the other hand, the first part of (Ba-Kr 2) is a lifting property for orderings from $\bar{K}$ to $K$, under certain circumstances.

A previous unrefereed version of this paper is [Pu1]. 


\section{NOTATIONS}

$\star$ An involution of a $\operatorname{ring} A$ is an element $\tau$ of the automorphism group Aut $(A)$ such that $\tau^{2}=\mathrm{id}_{A}$. In this paper we will assume, in addition, that involutions are not trivial, i.e., $\tau \neq \mathrm{id}_{A}$. If $\tau$ is an involution of $A$, then $A^{+}$ clenotes the subring of $A$ consisting of the fixed points of $A$; it is called the fixed ring of $A$.

* If $A_{1}, A_{2}$ are subfields of a field $A_{3}$, then $A_{1} \cdot A_{2}$ denotes the smallest subfield of $A_{3}$ containing $A_{1} \cup A_{2}$.

* Let $E$ be an algebraically closed field. Then $\pm i_{E}$ denote the solutions of the equation $X^{2}+1=0$ in $E$. When $E$ is understood, we will simply write $\pm i$. In this paper, $\epsilon$ is always either $i$ or $-i$.

$\star E^{a}$ denotes the algebraic closure of a field $E$.

* We fix a field extension $R \subseteq K$ with $R$ real. Then $K$ has zero characteristic, obviously. Thus, there exist field morphisms $j: K \rightarrow C$ with $C$ some algebraically closed field of zero characteristic and such a field $C$ possesses involutions $\tau$ that fix $j(R)$ pointwise (see $[\mathrm{S}]$ to learn about the abundance of involutions in zero characteristic algebraically closed fields). Let $\epsilon \in\left\{ \pm i_{C}\right\}$ and take triplets $(j, \tau, \epsilon)$ such that $C$ is algebraic over $j(R) \cdot \tau j(R)$. Say two triplets $\left(j_{s}, \tau_{s}, \epsilon_{s}\right)$ with $s=1,2$ are equivalent if there exists a field isomorphism $f: C_{1} \rightarrow C_{2}$ such that $f\left(\epsilon_{1}\right)=\epsilon_{2}, j_{2}=f j_{1}$ and $\left.f\right|_{C_{1}^{\tau_{1}}}: C_{1}^{\tau_{1}} \rightarrow C_{2}^{\tau_{2}}$ is a field isomorphism (order-preserving, a fortiori). The points of the complex spectrum of $K$ over $R, \operatorname{Spec}_{\mathrm{c}}(K / R)$, are equivalence classes $[j, \tau, \epsilon]$ of such triplets. We have just explained that $\operatorname{Spec}_{\mathrm{c}}(K / R)$ is non-empty, provided that $R$ is reai. The complex spectrum has been studied by the anthor in [Pu3] and earlier in [Pu2]. If $K$ is also real then $\operatorname{Spec}_{\mathrm{c}}(K / R)$ extends $\operatorname{Spec}_{r}(K)$ in the following sense. For each ordering $\beta$ of $K$ consider the real closure $F$ of $(K, \beta)$. Then $F^{a}$ has a unique involution $\tau$ fixing $F$ and having $\tau(i)=-i$. Thus $\beta \mapsto[j, \tau, i]$ defines a (canonical) mapping $\Upsilon: \operatorname{Spec}_{\mathrm{r}}(K) \rightarrow \operatorname{Spec}_{\mathrm{c}}(K / R)$, where $j: K \rightarrow F^{a}$ is the field embedding considered above. (In this paper we only use the existence of $\Upsilon$. However, as one would expect, $\Upsilon$ is continuous and a homeomorphism onto the image, provided that the spectra are endowed with their usual topologies; see 2.5, 2.6 and $2.7[\mathrm{Pu} 3])$. 
Lot $R \subseteq K$ be a ficld extension with $R$ real. In this paper we will deal with just a point in $\operatorname{Spec}_{\mathrm{c}}(K / R)$ at a time, so that the notation $z=[j, \tau, \epsilon]$ can and will often be simplified to $z=[K \subseteq C, \tau, \epsilon]$; this conveys that $j$ is the identity on $K$ and each element of $K$ is identified with its image in $C$. We have then $R \subseteq C^{\tau}$ (although the notation does not make this explicit).

First we observe that $z=[K \subseteq C, \tau, \epsilon]$ induces a point in $\operatorname{Spec}_{\mathrm{r}}\left(K \cap C^{\tau}\right)$, namely the ordering induced by the real closed field $C^{\tau}$ on $K \cap C^{\tau}$. Let us denote it by re( $(z)$. Note that $K \subseteq C^{\tau}$ iff $K=K \cap C^{\tau}$ iff $\Upsilon \operatorname{re}(z)=z$, with $\Upsilon$ as in the notations. We may say that the point $z$ is real if any of the equivalent conditions above hold. Note that $\operatorname{Spec}_{\mathrm{c}}(K / R)$ contains real points iff $K$ is real.

Given $z=[K \subseteq C, \tau, \epsilon]$ and $a \in K$ set $\mathrm{N}_{\tau}(a):=a \tau(a) \in C^{\tau}$ and call it the $\tau$-norm of $a$. In fact, $\mathrm{N}_{\tau}(a)$ is non-negative, being a sum of squares in $C^{\tau}$. Indeed $\mathrm{N}_{\tau}(a)=\left(\frac{a+\tau(a)}{2}\right)^{2}+\left(\frac{a-\tau(a)}{2 i}\right)^{2}$. In general, $\mathrm{N}_{\tau}(a) \notin K$, since in general $\tau(K) \nsubseteq K$.

1. Definitions. Let $R \subseteq K$ be a field extension with $R$ real, $B$ a valnation ring of $K$ and $S \subseteq K$ any subset. Let $z=[K \subseteq C, \tau, \epsilon]$ belong to Sper. $(K / R)$.

(a) $B$ is called $z$-conver (in $K$ ) if for all $a \in K, b \in B$, the condition $\mathrm{N}_{\tau}(a) \leq \mathrm{N}_{\tau}(b)$ implies $a \in B$.

(b) $S$ is called $\tau$-invariant if $\tau(S)=S$, (equivalently, if $\tau(S) \subseteq S$ ).

Though (a) has been defined using a specific representative of $z$, it is not hard to show that this definition is independent of the representative chosen.

Note that $\mathrm{N}_{\tau}(a)=a^{2}$, for all $a \in K$, when $z$ is a real point. Thus, both refinitions (real and complex) of convexity agree in this case.

2. Remarks. (a) Assume that $B$ is $\tau$-invariant. Then all $m_{B}, U_{B}$ and $K$ are $\tau$-invariant. Call $\tau^{\prime}$ the restriction of $\tau$ to $K$. Then $\tau^{\prime}=\operatorname{id}_{K}$ iff $K \subseteq C^{\tau}$ iff $z$ is real.

(b) Assume that $K$ is algebraically closed, $\tau$ is an involution of $K$ and $B$ is $\tau$-invariant. Then the mapping defined by $\bar{\tau}\left(x+m_{B}\right)=\tau(x)+m_{B}$, for all $x \in B$, is an involution of $\bar{K}$ (note that $i \in U_{B}$, since $B$ is integrally closed, whence $\bar{\tau}\left(i+m_{B}\right)=-i+m_{B} \neq i+m_{B}$, so that $\left.\bar{\tau} \neq \mathrm{id}_{\bar{K}}\right)$. In particular, $\lambda_{B} \tau=\bar{\tau} \lambda_{B}$. Conversely, if $\sigma$ is any involution of $\bar{K}$ such that $\lambda_{B} \tau=\sigma \lambda_{B}$, then $B$ is $\tau$-invariant and $\sigma=\bar{\tau}$.

(c) The condition $\lambda_{B} \tau=\sigma \lambda_{B}$ obviously implies $\lambda_{B} \mathrm{~N}_{\tau}=\mathrm{N}_{\sigma} \lambda_{B}$. 
3. Proposition. Let $R \subseteq K$ be a field extension with $R$ real, $z=[K \subseteq$ $C, \tau, \epsilon]$ a point in $\operatorname{Spec}_{c}(K / R)$ and $B$ a $z$-convex valuation ring of $K$. Then the following hold true:

(i) $B$ is finite over $\mathbb{Q}$ and char $\bar{K}=\mathbf{0}$,

(ii) $B \cap C^{\tau}$ is a $\operatorname{re}(z)$-convex valuation ring of $K \cap C^{\tau}$,

(iii) if $K$ is $\tau$-invariant then $B$ is $\tau$-invariant.

Proof. (i) We have $n^{-2}=\mathrm{N}_{\tau}\left(n^{-1}\right)<\mathrm{N}_{\tau}(1)=1$, hence $n^{-1} \in B$, for all $1<n \in \mathbb{N}$, whence $\mathbb{Q}$ is a subring of $B$ and of $\bar{K}$.

(ii) Immediate.

(iii) $\tau(K)=K$ implies $\tau(B)=B$ simply because $\tau(a)^{\circ} \in K, B$ is $z$-convex and $\mathrm{N}_{\tau}(\tau(a))=a \tau(a)=\mathrm{N}_{\tau}(a)$, for all $a \in B$.

4. Proposition. Let $R \subseteq K$ be a field extension with $R$ real, $z=[K \subseteq$ $C, \tau, \epsilon]$ a point in $\operatorname{Spec}_{\mathrm{c}}(K / R)$ and $B$ a valuation ring of $K$. If $B$ is $\tau$-invariant and $B \cap C^{\tau}$ is re $(z)$-convex, then $B$ is $z$-convex.

Proof. Suppose that $a \in K, b \in B$ and $\mathrm{N}_{\tau}(a) \leq \mathrm{N}_{\tau}(b)$. Since $\tau(B)=B$, then $\mathrm{N}_{\tau}(b)=b \tau(b)$ lies in $B \cap C^{\tau}$, whence $\mathrm{N}_{\tau}(a)=a \tau(a)$ also lies in $B \cap C^{\tau}$, by re(z)-convexity. Moreover if $a \notin B$ then $a^{-1} \in m_{B}$, whence $\tau(a)=$ $a^{-1} a \tau(a) \in m_{B} \subseteq B=\tau(B)$. Applying $\tau$ we get $a \in B$, a contradiction. Therefore, $a \in B$ holds, as was to be shown.

Since we will only be interested in $z$-convex valuation rings, then we will restrict ourselves to valuation rings finite over $\mathbb{Q}$, by Proposition 3 (i).

Let $z=[K \subseteq C, \tau, \epsilon]$ be a point in $\operatorname{Spec}_{\mathrm{c}}(K / R)$ and set $\mathcal{H}:=\{x \in$ $K: \mathrm{N}_{\tau}(x) \leq n^{2}$, some $\left.n \in \mathbb{N}\right\}$. Then $\mathcal{H}$ is a $z$-convex valuation ring in $K$. Indeed, it is easy to verify that $\mathcal{H}$ is closed under products and sums, using that $\mathrm{N}_{\tau}(x+y) \leq 4 \max \left\{\mathrm{N}_{\tau}(x), \mathrm{N}_{\tau}(y), \mathrm{N}_{\tau}(x \tau(y)), 1\right\}$, for all $x, y \in K$. It is also clear that $x \in K \backslash \mathcal{H}$ implies $x^{-1} \in \mathcal{H}$.

5. Proposition. Let $R \subseteq K$ be a field extension with $R$ real, $z=[K \subseteq$ $C, \tau, \epsilon]$ a point in $\operatorname{Spec}_{\mathrm{c}}(K / R)$. If $B$ is any valuation ring of $K$ finite over $\mathbb{Q}$, then the following are equivalent:
(a) $B$ is $z$-convex in $K$,
(b) $m_{B}$ is $z$-convex in $B$,
(c) $\mathcal{H} \subseteq B$.

Proof. (a) $\Rightarrow$ (b) Let $a \in B, b \in m_{B}$ and suppose $\mathrm{N}_{\tau}(a) \leq \mathrm{N}_{\tau}(b)$. If $b=0$ then $0=\mathrm{N}_{\tau}(a)=a=\tau(a)$, whence $a$ belongs to $m_{B}$. If $a \neq 0$ then 
$b \neq 0, \mathrm{~N}_{\tau}\left(b^{-1}\right) \leq \mathrm{N}_{\tau}\left(a^{-1}\right)$ and $b^{-1} \notin B$, whence $a^{-1} \notin B$, by $z$-convexity of $B$. Thus, $a$ belongs to $m_{B}$.

(b) $\Rightarrow$ (c) Let $x \in K$ be such that $\mathrm{N}_{\tau}(x) \leq n^{2}$, for some $n \in \mathbb{N}$. Suppose $x \notin B$. Then $n \neq 0, x^{-1} \in m_{B}$ and $n^{-2} \leq \mathrm{N}_{\tau}\left(x^{-1}\right)$ whence $n^{-1}$ belongs to $m_{B}$, by $z$-convexity of $m_{B}$ and finiteness of $B$ over $\mathbb{Q}$. It follows that 1 belongs to $m_{B}$, which is absurd.

(c) $\Rightarrow$ (a) Let $a \in K, b \in B$ and suppose $\mathrm{N}_{\tau}(a) \leq \mathrm{N}_{\tau}(b)$. If $b=0$ then $0=\mathrm{N}_{\tau}(a)=a=\tau(a)$. If $b \neq 0$ then $\mathrm{N}_{\tau}\left(a b^{-1}\right) \leq 1$ whence $a b^{-1} \in \mathcal{H} \subseteq$ $B$ and so $a=\left(a b^{-1}\right) b \in B$.

6. Definitions. Let $R \subseteq K$ be a field extension with $R$ real. Consider $z=[K \subseteq C, \tau, \epsilon] \in \operatorname{Spec}_{\mathrm{c}}(K / R)$. Let $E, F$ be intermediate fields with $R \subseteq E \subseteq K \subseteq F \subseteq C$.

(a) $C$ is algebraic over $K \cdot \tau(K)$ whence also algebraic over $F \cdot \tau(F)$. We drfine the extension of $z$ to $F$ to be the point $[F \subseteq C, \tau, \epsilon] \in \operatorname{Spec}_{\mathrm{c}}(F / R)$ and donote it by $\operatorname{ext}(z, F)$.

(b) Let $C^{\prime}$ be the algebraic closure of $E \cdot \tau(E)$ inside $C$. Clearly $\epsilon$ belongs to $C^{\prime}$. Now, for every $a \in C^{\prime}, \tau(a)$ is a root of the image by $\tau$ of the minimal polynomial of a over $E \cdot \tau(E)$, whence $\tau(a)$ belongs to $C^{\prime}$. It follows that the Iestriction $\tau^{\prime}$ of $\tau$ to $C^{\prime}$ is an involution (if $\tau^{\prime}=\mathrm{id}_{C^{\prime \prime}}$ then $C^{\prime}$ would embed into a real closed field, contradicting that $C^{\prime}$ is algebraically closed). The point $\left[E \subseteq C^{\prime}, \tau^{\prime}, \epsilon\right]$ belongs to $\operatorname{Spec}_{\mathrm{c}}(E / R)$ and is called the restriction of $z$ to $E$ and denoted $\operatorname{res}(z, E)$.

The extensions of $z$ to all intermediate fields $F$ are dominated by the extension to $C$, in the sense that $\operatorname{ext}(z, F)=\operatorname{res}(\operatorname{ext}(z, C), F)$. For short, write $z^{\dagger}=\operatorname{ext}(z, C)$.

The proof of the following result is immediate.

7. Proposition. Let $R \subseteq E \subseteq K$ be a field extension with $R$ real. Let $z=[K \subseteq C, \tau, \epsilon]$ be a point in $\operatorname{Spec}_{c}(K / R)$ and $B \subseteq K$ a valuation ring. Consider $w=\operatorname{res}(z, E) \in \operatorname{Spec}_{c}(E / R)$. If $B$ is $z$-convex then $B \cap E$ is w-convex.

8. Definition and Proposition. Let $R \subseteq K \subseteq C$ be a field extension with $R$ real and $C$ algebraically closed. Let $B$ be a subring of $K$ and $\tau$ an involution of $C$. The set

$$
\mathcal{O}_{\tau}(B):=\left\{x \in C: \mathrm{N}_{\tau}(x) \leq \mathrm{N}_{\tau}(b), \text { some } b \in B\right\}
$$


is called the $\tau$-convex hull of $B$. It is a $w$-convex, $\tau$-invariant valuation ring of $C$, where $w=[C \subseteq C, \tau, \epsilon] \in \operatorname{Spec}_{\mathrm{c}}(C / R)$. In addition, if $B$ is a valuation ring of $K$ then $B$ is $z$-convex if and only if $\mathcal{O}_{\tau}(B)$ is an extension of $B$, i.e., $\mathcal{O}_{\tau}(B) \cap K=B$.

Proof. It is easy to verify that $\mathcal{O}_{\tau}(B)$ is closed under products and sums, using that $\mathrm{N}_{\tau}(x+y) \leq 4 \max \left\{\mathrm{N}_{\tau}(x), \mathrm{N}_{\tau}(y), \mathrm{N}_{\tau}(x \tau(y)), 1\right\}$, for all $x, y \in C$. It is straightforward to show that $\mathcal{O}_{\tau}(B)$ is a $w$-convex valuation ring. It is $\tau$-invariant just because $\mathrm{N}_{\tau}(x)=\mathrm{N}_{\tau}(\tau(x))$. Finally, it is immediate to realize that $\mathcal{O}_{\tau}(B) \cap K=B$ if and only if $B$ is $z$-convex.

From here on, we set $f(\infty)=\infty$, for any map $f$.

9. Construction: Complex $(\mathrm{Ba}-\mathbf{K r} \mathbf{1})$. Let $B \subseteq K$ be a valuation ring finite over $\mathbb{Q}$ and $z=[K \subseteq C, \tau, \epsilon] \in \operatorname{Spec}_{\mathrm{c}}(K / R)$. Write $\bar{R}$ for the residue class field of the valuation $\operatorname{ring} B \cap R$. Suppose that $B$ is $z$-convex. Then $B \cap R$ is convex in $R$ with respect to the ordering induced by $C^{\tau}$.

Easy case: Suppose $K=C$. Then $K$ is algebraically closed and $\tau$ invariant. By Proposition 3 (iii), $B$ is $\tau$-invariant. By Remarks 2 (b), $\tau$ induces an involution $\bar{\tau}$ on $\bar{K}$ such that $\lambda_{B} \tau=\bar{\tau} \lambda_{B}$. Moreover $\bar{K}$ is algebraically closed, by $[\mathrm{PC}]$ lemma 17 , page 47 . The fixed field $\bar{K}^{\bar{\tau}}$ contains $\bar{R}$, since $R \subseteq K^{\tau}$. In this situation, we say that $z$ and $B$ induce the point $z_{B}:=\left[\bar{K} \subseteq \bar{K}, \bar{\tau}, \lambda_{B}(\epsilon)\right] \in \operatorname{Spec}_{c}(\bar{K} / \bar{R})$. Such a point $z_{B}$ is unique in Spec $_{c}(\bar{K} / \bar{R})$ with the conditions $\lambda_{B} \tau=\bar{\tau} \lambda_{B}$ and $\lambda_{B}(\epsilon)$ appearing in the last entry (clearly $\left.\lambda_{B}(\epsilon) \in\{ \pm i\}\right)$.

General case: If $K$ is not necessarily equal to $C$, then extend $z$ to $z^{\dagger} \epsilon$ $\operatorname{Spec}_{\mathrm{c}}(C / R)$ and consider $H:=\mathcal{O}_{\tau}(B)$, which is a $z^{\dagger}$-convex valuation ring of $C$ extending $B$, using Definitions 6 and 8 . To $z^{\dagger}$ and $H$ we apply the construction of the previous easy case, thus obtaining an induced point $\left(z^{\dagger}\right)_{H}$. It lies in $\operatorname{Spec}_{\mathrm{c}}(\bar{C} / \bar{R})$, being $\bar{C}$ (respectively, $\bar{R}$ ) the residue class field of $H$ (respectively, of $H \cap R=B \cap R$ ).

The condition $H \cap K=B$ conveys inclusions $B \subseteq H$ and $m_{B} \subseteq m_{H}$. They induce a field embedding $l: \bar{K} \rightarrow \bar{C}$. In particular, the place $\lambda_{H}$ : $C \rightarrow \bar{C} \cup\{\infty\}$ satisfies $l \lambda_{B}=\left.\lambda_{H}\right|_{B}$. If we identify each element of $\bar{K}$ with its image by $l$ then $\lambda_{H}$ is an extension of $\lambda_{B}$ to $C$ and we will simply write $\lambda$ for either $\lambda_{B}$ or $\lambda_{H}$. Now, the field extension $\bar{K} \cdot \bar{\tau}(\bar{K}) \subseteq \bar{C}$ is algebraic, since $K \cdot \tau(K) \subseteq C$ is algebraic. We have a point $\operatorname{res}\left(\left(z^{\dagger}\right)_{H}, \bar{K}\right)$ that will 
be denoted $z_{B}$ and called point induced by $z$ and $B$ in. $\operatorname{Spec}_{c}(\bar{K} / \bar{R})$, namely, $z_{B}=\left[\bar{K} \subseteq \bar{C}, \bar{\tau}, \lambda_{B}(\epsilon)\right]$.

Note that $H$ is $\tau$-invariant. Thus the equality $\lambda \tau=\bar{\tau} \lambda$ holds and it follows that $\lambda \mathrm{N}_{\tau}=\mathrm{N}_{\bar{\tau}} \lambda$.

How does this fit with the real $(B a-K r 1)$ ? $\mathrm{re}\left(z^{\dagger}\right)$ is nothing but the unique ordering of $C^{\tau}$ and $\operatorname{re}\left(\left(z^{\dagger}\right)_{H}\right)$ is the unique ordering of $\bar{C} \bar{C}^{\tau}$. Thus $\mathrm{re}\left(z_{13}\right)$ is the ordering induced on $\bar{K} \cap \bar{C}^{\bar{\tau}}$. Then the ordering $\overline{\mathrm{re}(z)}$ given by the real $(B a-K r 1)$ coincides with $\operatorname{re}\left(z_{B}\right)$. Indeed, given $x \in U_{B} \cap C^{\tau}$, we have $\lambda(x)>\frac{1 \mathrm{e}(z)}{0} 0$ iff $x>_{\mathrm{re}(z)} 0$ iff $x=a^{2}$, for some $a \in C^{\tau} \backslash\{0\}$. This implies that $0 \neq \lambda(x)=\lambda(a)^{2}$ and this holds iff $\lambda(x)>_{\operatorname{re}(z B)} 0$.

10. Definition. Let $R \subseteq K$ be a field extension with $R$ real, $B$ a valuation ring of $K$. Suppose that the residue class field $\bar{R}$ of $B \cap R$ is a real subfield of $\bar{K}$. If $w=[\bar{K} \subseteq D, \sigma, \epsilon]$ belongs to $\operatorname{Spec}_{\mathrm{c}}(\bar{K} / \bar{R})$, then a lifting of $w$ via $\lambda_{B}$ is a point $z$ in $\operatorname{Spec}_{\mathrm{c}}(K / R)$, such that $B$ is $z$-convex and $z_{B}=w$.

The generalization of (Ba-Kr 2) to the complex spectrum claims that liftings exist. It will follow easily from the next result, the proof of which is due to A. Prestel.

11. Proposition. Let $R \subseteq K$ be a field extension with $R$ real, $B \subseteq K$ a valuation ring, $D$ an algebraically closed extension of $\bar{K}$ with char $D=0, \sigma$ an involution of $D$. Suppose that the residue class field $\bar{R}$ of $B \cap R$ satisfies $\bar{R} \subseteq D^{\sigma}$. Then there exist an algebraically closed extension $C^{*}$ of $K$ and an involution $\tau^{*}$ of $C^{*}$ such that for some extension $H^{*}$ of $B$ to $C^{*}$ we have

(a) $H^{*}$ is $\tau^{*}$-invariant (and thus $\tau^{*}$ induces an involution $\overline{\tau^{*}}$ of $\overline{C^{*}}$ ),

(b) $D \subseteq \overline{C^{*}}$ and $\overline{\tau^{*}}$ extends $\sigma$,

(c) $R \subseteq\left(C^{*}\right)^{\tau^{*}}$.

Proof. It is well known that there exists an extension $v^{a}$ of $v_{B}$ to the algebraic closure $K^{a}$ and that the value group of $v^{a}$ is the divisible hull $\Delta$ of $\Gamma_{B}$. There exists an embedding of $R$ into $D^{\sigma}((\Delta))$ as valued fields (see [Ka], page 318). Since $D^{\sigma}((\Delta))$ is real closed then some real closure $E$ of $R$ inside $K^{a}$ embeds into $D^{\sigma}((\Delta))$ as ordered compatibly-valued fields, by [PC] page 118 , theorem 27 . Write $C=D((\Delta))$, denote by $u$ the canonical valuation of $C$ and by $\tau$ the canonical involution in $C$ extending $\sigma$ (so that $\bar{\tau}=\sigma$ holds and $\left.C^{\tau}=D^{\sigma}((\Delta))\right)$. The embedding $\left(E,\left.v^{a}\right|_{E}\right) \hookrightarrow\left(C^{\tau}, u \check{C}^{\tau}\right)$ has a canonical extension to some embedding of $E(i)$ into $C$ as valued fields. So we have $\left(E(i), v^{a} \check{E}(i)\right) \stackrel{\varphi}{\rightarrow}(C, u)$, for some embedding $\varphi$. We would like 
to extend $\varphi$ to some embedding of $\left(K^{a}, v^{a}\right)$ into some extension $\left(C^{*}, v^{*}\right)$ of $(C, v)$.

By general model theory (see $[\operatorname{Pr} 1]$ page 125 or $[\operatorname{Pr} 2]$ page 47$)$, there exists a card $(K)^{+}$-saturated elementary extension $\left(C^{*}, u^{*}, \tau^{*}\right)$ of the structure $(C, u, \tau)$. Now, all the conditions in $[\mathrm{K}-\mathrm{P}]$, claim in page 192 , are satisfied for $K_{1}=E(i), K_{2}=K^{a}$ and $K^{*}=C^{*}$. Indeed, $E(i)$ and $C^{*}$ are obviously henselian valued fields. Their residue class fields satisfy $\overline{E(i)} \subseteq \overline{K^{a}} \subseteq \overline{C^{*}}$ and $\overline{E(i)}$ is algebraically closed (in $\overline{K^{a}}$ ). And their value groups satisfy $v^{a}(E(i)) \subseteq \Delta \subseteq u^{*}\left(C^{*}\right)$ and the residue class group $\Delta / v^{a}(E(i))$ is torsion-free, since $v^{a}(E(i))$ is divisible. Thus, by $[\mathrm{K}-\mathrm{P}], \varphi$ extends to $\left(K^{a}, v^{a}\right) \stackrel{\varphi}{\hookrightarrow}\left(C^{*}, u^{*}\right)$.

Clearly, $\tau^{*}$ is an involution extending $\tau$ and thus $\varphi(R) \subseteq\left(C^{*}\right)^{\tau^{*}}$. If we now identify each element of $K^{a}$ with its image by $\varphi$ then (c) holds.

The clefinition of $\tau$ implies that $\rho \tau=\sigma \rho$, where $\rho$ denotes the canonical place of $C$. Therefore the associated valuation ring $H \subseteq C$ is $\tau$-invariant, by Remarks 2 (b). This means that $u(x) \geq 0$ implies $u \tau(x) \geq 0$, for $x \in C$. If $H^{*} \subseteq C^{*}$ denotes the valuation ring associated to $u^{*}$ then (a) follows, since the extension is elementary.

The condition $H^{*} \cap C=H$ conveys inclusions $H \subseteq H^{*}$ and $m_{H} \subseteq m_{H^{*}}$. They induce a field embedding $l: D \rightarrow \overline{C^{*}}$. If we identify each element of $D$ with its image by $l$ then $\left.\overline{\tau^{*}}\right|_{D}=\bar{\tau}=\sigma$ and (b) holds.

Note that for a power series to be saturated, the value group must be saturated. For instance, $C=\mathbb{C}((\mathbb{Q}))$ is not saturated.

12. Corollary: Complex (Ba-Kr 2). Let $R \subseteq K$ be a field extension with $R$ real, $B$ a valuation ring of $K$. Suppose that the residne class field $\bar{R}$ of $B \cap R$ is a real subfield of $\bar{K}$. Then for each $w=[\bar{K} \subseteq D, \sigma, \epsilon] \in \operatorname{Spec}_{c}(\bar{K} / \bar{R})$ there exists a lifting of $w$ via $\lambda_{B}$.

Proof. We apply Proposition 11 and obtain $C^{*}, \tau^{*}$ and $H^{*}$, with the notations of the previous proof. Take $\delta \in \rho^{-1}(\epsilon) \cap\{ \pm i\}$ and consider the points $z^{*}=\left[C^{*} \subseteq C^{*}, \tau^{*}, \delta\right] \in \operatorname{Spec}_{\mathbf{c}}\left(C^{*} / R\right)$ and $z=\operatorname{res}\left(z^{*}, K\right) \in \operatorname{Spec}_{\mathrm{c}}(K / R)$. We have $z=\left[K \subseteq C^{\prime}, \tau^{\prime}, \delta\right]$ where $C^{\prime}$ is the algebraic closure of $K \cdot \tau^{*}(K)$ in $C^{*}$ and $\tau^{\prime}$ is the restriction of $\tau^{*}$ to $C^{\prime}$. We must verify that $B$ is $z$-convex and $z_{B}=w$.

Using the notations in the proof of Proposition 11, recall that $C^{\tau}$ is an ordered compatibly-valued field, which implies that $H \cap C^{r}$ is a convex valuation ring of $C^{\top}$. By Proposition $4, H$ is $t$-convex, where $t=[C \subseteq$ 
$C, \tau, \delta]$. This means that $u(b) \geq 0, b \tau(b)-a \tau(a)=c^{2}$ and $c=\tau(c)$ imply $u(a) \geq 0$, for $a, b, c \in C$. Since the extension is elementary then $H^{*}$ is $z^{*}$-convex. Thus $B=H^{*} \cap K$ is $z$-convex, by Proposition 7 .

Finally, $z_{B}=w$ because the extensions of $z$ to $C^{\prime}$ (respectively, of $z_{B}$ and $w$ to $\overline{C^{\prime}}$ ) are dominated by $z^{*}$ (respectively, by $\left(z^{*}\right)_{H^{*}}$.

The proof of the existence of liftings of $w$, in the case $R=\mathbb{Q}$ is simpler than the proof given above. It uses a version of Proposition 11, which does not call for saturated structures. We just embed $K$ into $D((\Delta))$ as valued ficlds and, of course, this embedding maps $\mathbb{Q}$ into $D^{\sigma}((\Delta))$.

The question of determining all the liftings of $w$ via $\lambda_{B}$ does not seem to follow easily from the above.

It seems possible to derive a complex ( $\mathrm{Ba}-\mathrm{Kr} 2)$ from 2.12 in $[\mathrm{H}]$.

\section{ACKNOWLEDGMENTS}

This paper arises from a question posed by G. Brumfiel to the author. Nany discussions (some through e-mail) with him helped the author to clarify various statements and proofs. When most of the work was already done, the anthor had a proof of the complex second Baer-Krull theorem only for $R=\mathbb{Q}$, but lacked one for an arbitrary real field $R$. A discussion with A. Prestel and a later private communication from him provided that proof. The author is very much indebted to both of them.

Parts of this paper were worked on during the Special Semester in Real Algebraic Geometry and Ordered Algebraic Structures (RAGOS), at LSU, in Spring of 1996. Special aknowledgements are due to the organizers of the scmester C. Delzell, J. Madden and S. Woodward, for making our stay a very pleasant one.

\section{REFERENCES}

[Ba] R. Baer, Über nicht-archimedisch geordnete Körper (Beiträge zur Algebra 1). Sitz. Ber. der Heidelberger Akademie, 8. Abhandl., (1927), 3-13.

[Br] R. Brown, Real places and ordered fields, Rocky Mountain J. Math. 1, (1971), 633-636.

[H] R. Huber, Spezialisierungen im Reellen Spectrum zu einer Komplexen Varietät, Geom. Dedic. 38, (1991), 309-327. 
[Ka] I. Kaplansky, Maximal fields with valuations, Duke Math. J. 9, (1942), 303-321.

[Kr] W. Krull, Allgemeine Bewertungstheorie, J. reine angew. Math. 167, (1932), 160-196.

[K-P] F.V. Kuhlmann, A. Prestel, On places of algebraic function fields, J. reine angew. Math. 353, (1984), 181-195.

[L] S. Lang, The theory of real places, Ann. of Math. 57 (1953), 378-391.

[Pr1] A. Prestel, Einführung in die Mathematische Logik und Modeltheorie, Vioweg Studium, Braunschweig/Wiesbaden, 1986.

[Pr2] A. Prestel, Model theory for the real algebraic geometer, Instituti Editoriali e Poligrafici Internazionali, Pisa-Roma, 1998.

[PC] S. Priess-Crampe, Angeordnete Structuren: Gruppen, Körper, projective Ebenen, Ergebnisse der Math. 98, Springer-Verlag, Berlin-HeidelbergNew York, 1983.

[Pu1] M.J. de la Puente, A complex version of the Baer-Krull theorems, Prepubications, Equipe de Logique, Sćminaire de Structures Algébriques Ordomnées, no. 61, Université Paris VII, (1997).

[Pu2] M.J. de la Puente, The complex spectrum of a ring, Prepubications, Equipe de Logique, Séminaire de Structures Algébriques Ordonnées, no. 61, Université Paris VII, (1997).

[Pu3] M.J. de la Puente, The complex spectrum of a ring, in Cont. Math. AMS, Proceedings of the RAGOS special semester, C. Delzell and J. Madden eds., (2000), 235-249.

[S] B. Schnor, Involutions in the group of automorphisms of an algebraically closed field, J. of Alg. 152, (1992), 520-524. 